THE ASTROPHYSICAL JOURNAL, 298:275-280, 1985 November 1

(C) 1985. The American Astronomical Society. All rights reserved. Printed in U.S.A.

\title{
INFRARED PHOTOMETRY OF THE NEBULOSITY AROUND QUASARS
}

\author{
G. Neugebauer, K. Matthews, B. T. Soifer, and J. H. Elias \\ Palomar Observatory, California Institute of Technology \\ Received 1985 February 11; accepted 1985 April 26
}

\begin{abstract}
Measurements of the nebulosity around quasars have been carried out in the near-infrared. The colors of the host galaxies found in these observations are consistent with those of normal galaxies. The luminosities are also similar to those of normal galaxies but are slightly brighter than average.

Subject headings: galaxies: photometry — infrared: sources - quasars
\end{abstract}

\section{INTRODUCTION}

The existence of nebulosity around some quasars, commonly presumed to be evidence for the presence of host galaxies, has been known for several years. Hutchings et al. (1984) and Hutchings, Crampton, and Campbell (1984) have summarized optical imaging of some 75 quasars; host galaxies are resolved in all but seven of the quasars. Visual images of nebulosity around more limited selected samples of quasars have also been obtained by Wyckoff, Wehinger, and Gehren (1981), Stockton (1982), Tyson, Baum, and Kreidl (1982), Gehren et al. (1984), and Malkan, Margon, and Chanan (1984). Boroson and Oke (1982), Boroson, Oke, and Green (1982), Balick and Heckman (1983), and Boroson and Oke (1984) have obtained spectra of the nebulosity around several quasars. Both the spectroscopic and the imaging studies point strongly to the conclusion that the nebulosities represent host galaxies around the quasars. Many of the observations are consistent with the interpretation that the nebulosity represents spiral galaxies, but there is no overwhelming consensus that all host galaxies are spiral galaxies.

In this paper, near-infrared observations of the nebulosity around 16 quasars are reported. The purpose of the observations was to estimate the contribution that such nebulosity could make to the observed energy distributions of quasars and to determine whether the infrared properties of the host galaxies associated with quasars were in any way unique.

\section{OBSERVATIONS AND RESULTS}

All the observations were carried out at the $5 \mathrm{~m}$ Hale telescope of the Palomar Observatory. A solid nitrogen cooled $\mathrm{InSb}$ detector was used at the Cassegrain focus of the $\mathrm{f} / 70$ chopping secondary. Photometry with filters defining the standard $J$ (center wavelength $\left.=\lambda_{0}=1.3 \mu \mathrm{m}\right), H\left(\lambda_{0}=1.65 \mu \mathrm{m}\right)$, and $K\left(\lambda_{0}=2.2 \mu \mathrm{m}\right)$ magnitudes was done using standard stars given by Elias et al. (1982). In all cases, the reference beam positions were $15^{\prime \prime}$ to the north and south of the object being measured.

Two methods of observation were used to measure the nebulosity. The majority of the measurements were made using an annular diaphragm with an opaque inner disk of $5^{\prime \prime}$ diameter centered within a $10^{\prime \prime}$ diameter circular diaphragm to measure the nebulosity. Separate measurements using a $5^{\prime \prime}$ diameter circular diaphragm were used to obtain the flux in the central $5 "$. For four of the observations, the flux measured through a circular diaphragm $5^{\prime \prime}$ in diameter and centered on the quasar was compared with the flux measured through diaphragms either $10^{\prime \prime}$ or (in 1982 December) $11^{\prime \prime}$ in diameter. These measurements, as can be seen in Table 1, are of somewhat lower accuracy.

All the observations were carried out by alternating measurements of the quasar and of a nearby star of similar infrared brightness. Typically, the comparison star was within $60^{\prime \prime}$ of the quasar. The visual seeing in all cases was required to be better than $2^{\prime \prime}$ full width at half-maximum and the amount of spill into the annular region outside of $5^{\prime \prime}$ diameter for a centered star was typically about $3 \%$. Most of this spill appears to be due to scattering in the telescope and instrument rather than to atmospheric seeing, and was thus quite stable $( \pm 0.3 \%)$. Nebulosity in the $5^{\prime \prime}-10^{\prime \prime}$ annulus which is $\ll 3 \%$ of the quasar flux would not, however, be readily detectable. Typically, four separate sets of measurements on the quasar and on the nearby star were carried out at each wavelength on each night.

The journal of observations and the measured results are given in Table 1. The objects are identified by their truncated 1950 equatorial coordinates. The primary measurement is the ratio of the flux in the annulus to that in the central $5^{\prime \prime}\left(F_{a} / F_{c}\right)$. The flux in the annulus has been corrected for the spill from the central object by assuming the spill when the quasar was centered in the diaphragm was equal to that when the nearby star was centered. The 1982 observations have been corrected to a $10^{\prime \prime}$ diameter diaphragm assuming the surface brightness of the nebular component varies as radius ${ }^{-1}$. When the flux in the annulus was less than 3 times its uncertainty, the magnitude limit of 3 times the uncertainty is quoted in Table 1.

The uncertainties quoted in Table 1 are standard deviations derived from the internal consistency of the measurements only. They are purely a measure of the uncertainty in the measurement of the nebulosity relative to that of the quasar. The effects of variable seeing are included in these uncertainties, although the annular measurements of the comparison stars indicate that the dominant source of uncertainty is usually photon statistics in the measurement of the weak fluxes seen in for the quasar annuli. There is an additional uncertainty of $\sim 0.05$ mag associated with the absolute photometry of the quasar. Measurements of the annular flux on more than one night indicate that the measurements of brighter objects are accurate to about $10 \%$. This is true both for objects like $0833+770$ and $0906+487$, where the annular flux is roughly $20 \%-40 \%$ that of the quasar, and for objects like $1251+640$ and $1545+210$, where the annular flux is proportionately much less.

Since the system was calibrated with respect to point, rather than extended, sources, systematic uncertainties up to $\sim 5 \%$ 
TABLE 1

ObServed Flux Ratios and Magnitudes

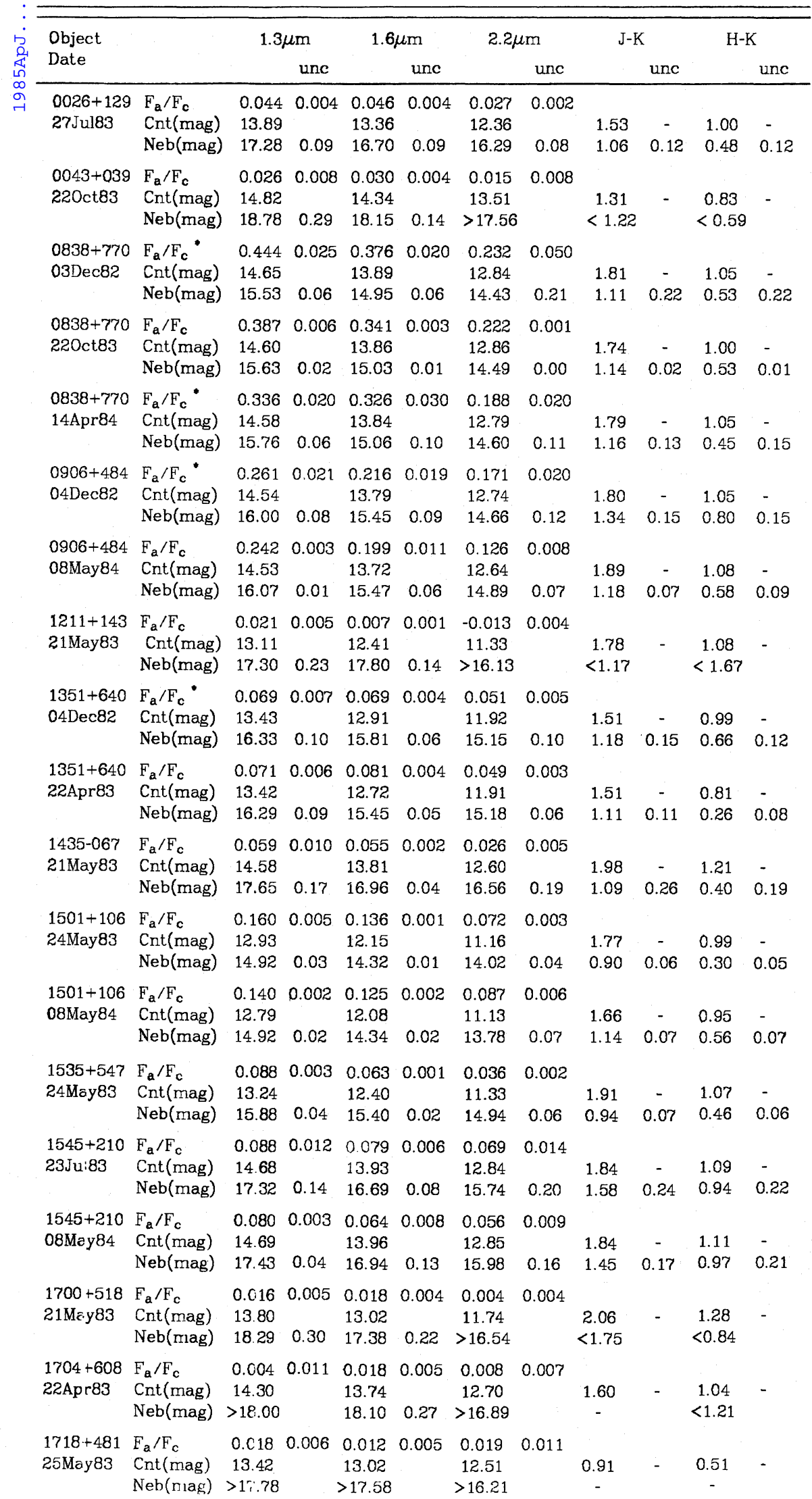




\begin{tabular}{|c|c|c|c|c|c|c|c|c|c|c|c|}
\hline \multirow{2}{*}{$\begin{array}{l}\text { Object } \\
\text { Date }\end{array}$} & & \multicolumn{2}{|c|}{$1.3 \mu \mathrm{m}$} & \multicolumn{2}{|c|}{$1.6 \mu \mathrm{m}$} & \multicolumn{2}{|c|}{$2.2 \mu \mathrm{m}$} & \multicolumn{2}{|c|}{$\mathrm{J}-\mathrm{K}$} & \multicolumn{2}{|c|}{$\mathrm{H}-\mathrm{K}$} \\
\hline & & & unc & & unc & & unc & & unc & & unc \\
\hline $2200+420$ & $\mathrm{~F}_{\mathrm{a}} / \mathrm{F}_{\mathrm{c}}$ & 0.170 & 0.002 & 0.169 & 0.004 & 0.101 & 0.005 & & & & \\
\hline \multirow[t]{2}{*}{ 25Мау83 } & Cnt(mag) & 12.74 & & 11.89 & & 10.99 & & 1.75 & - & 0.90 & - \\
\hline & Neb(mag) & 14.66 & 0.01 & 13.82 & 0.03 & 13.48 & 0.05 & 1.18 & 0.05 & 0.34 & 0.06 \\
\hline $2200+420$ & $\mathrm{~F}_{\mathrm{a}} / \mathrm{F}_{\mathrm{c}}$ & 0.129 & 0.003 & 0.114 & 0.001 & 0.084 & 0.001 & & & & \\
\hline \multirow[t]{2}{*}{ 26Ju:83 } & Cnt(mag) & 12.69 & & 11.87 & & 10.97 & & 1.72 & - & 0.90 & - \\
\hline & Neb(mag) & 14.91 & 0.02 & 14.23 & 0.01 & 13.66 & 0.01 & 1.25 & 0.03 & 0.57 & 0.02 \\
\hline \multirow{3}{*}{$\begin{array}{l}2200+420 \\
190 c t 83\end{array}$} & $\mathrm{~F}_{\mathrm{a}} / \mathrm{F}_{\mathrm{c}}$ & 0.103 & 0.001 & 0.093 & 0.001 & 0.073 & 0.002 & & & & \\
\hline & Cnt(mag) & 12.50 & & 11.67 & & 10.79 & & 1.71 & - & 0.88 & - \\
\hline & $\mathrm{Neb}$ (mag) & 14.97 & 0.01 & 14.25 & 0.01 & 13.63 & 0.03 & 1.34 & 0.03 & 0.62 & 0.03 \\
\hline \multirow{3}{*}{$\begin{array}{l}2209+184 \\
24 \text { May83 }\end{array}$} & $\mathrm{F}_{\mathrm{a}} / \mathrm{F}_{\mathrm{c}}$ & 0.333 & 0.016 & 0.305 & 0.005 & 0.259 & 0.004 & & & & \\
\hline & Cnt (mag) & 13.96 & & 13.27 & & 12.50 & & 1.46 & - & 0.77 & - \\
\hline & $\mathrm{Neb}$ (mag) & 15.15 & 0.05 & 14.56 & 0.02 & 13.97 & 0.02 & 1.19 & 0.05 & 0.59 & 0.02 \\
\hline \multirow{3}{*}{$\begin{array}{l}2209+184 \\
180 c t 83\end{array}$} & $\mathrm{~F}_{\mathrm{a}} / \mathrm{F}_{\mathrm{c}}$ & 0.347 & 0.011 & 0.289 & 0.007 & 0.230 & 0.006 & & & & \\
\hline & Cnt (mag) & 1397 & & 13.28 & & 12.49 & & 1.48 & - & 0.79 & - \\
\hline & $\mathrm{Neb}$ (mag) & 15.12 & 0.03 & 14.63 & 0.03 & 14.09 & 0.03 & 1.03 & 0.04 & 0.54 & 0.04 \\
\hline \multirow{3}{*}{$\begin{array}{l}2302+029 \\
25 \mathrm{Ju} 83\end{array}$} & $\mathrm{~F}_{\mathrm{a}} / \mathrm{F}_{\mathrm{c}}$ & 0.004 & 0.011 & 0.017 & 0.007 & 0.019 & 0.005 & & & & \\
\hline & Cnt(mag) & 14.84 & & 14.43 & & 13.80 & & 1.04 & - & 0.63 & - \\
\hline & Neb(mag) & $>18.54$ & & $>18.62$ & & 18.10 & 0.25 & $>0.44$ & & $>0.52$ & \\
\hline \multirow{3}{*}{$\begin{array}{l}2 乏 02+029 \\
190 \operatorname{ct} 83\end{array}$} & $\mathrm{~F}_{\mathrm{a}} / \mathrm{F}_{\mathrm{c}}$ & 0.012 & 0.006 & 0.006 & 0.004 & 0.013 & 0.006 & & & & \\
\hline & Cnt (mag) & 14.85 & & 14.45 & & 13.78 & & 1.07 & - & 0.67 & - \\
\hline & $\mathrm{Neb}$ (mag) & $>19.21$ & & $>19.25$ & & $>18.14$ & & - & & - & \\
\hline \multirow{3}{*}{$\begin{array}{l}2302+029 \\
220 c t 83\end{array}$} & $\mathrm{~F}_{\mathrm{a}} / \mathrm{F}_{\mathrm{c}}$ & 0.015 & 0.010 & 0.022 & 0.010 & 0.003 & 0.013 & & & & \\
\hline & Cnt(mag) & 14.88 & & 14.50 & & 13.90 & & 0.98 & - & 0.60 & - \\
\hline & Neb(mag) & $>18.69$ & & $>18.31$ & & $>17.42$ & & - & & - & \\
\hline
\end{tabular}

Note. - * Measured through two separate circular diaphragms. The rest were measured using an annulus of $5^{\prime \prime}$ inner diameter and $10^{\prime \prime}$ outer diameter.

may be present in the measurements of colors. Measurements of beam profiles indicate that these effects, if present, would make the colors appear redder than they actually are. It is possible that there are some systematic effects involved in centering the quasars and comparison star in the annulus as a result of their different visual brightness or colors which would lead to spurious detections of nebulosity. Estimates place this effect at less than $1 \%$ of the flux level of the quasar.

The quasars were selected for observation primarily on the basis of brightness, low redshift, and availability. Some were also chosen because optical and infrared measurements of the quasars made with diaphragms of different diameters gave apparently discordant results; see, e.g., Neugebauer et al. (1979). All but PG $0906+484$ and $2200+420$ (BL Lac) are included in the Palomar Bright Quasar Survey (Schmidt and Green 1983). The object PG $0906+484$ belongs to the larger Palomar-Green Survey but was omitted from the Palomar Bright Quasar Survey because it was fainter than the completeness limit in its area.

Two of the quasars listed in Table 1, 0906+484 and $1351+640$, have been observed in the $R$ and $B$ optical passbands by Hutchings, Crampton, and Campbell (1984). In both, there is clear optical evidence for host galaxies with generally circular appearance. A red plate of the quasar $1700+518$ by these same authors indicated no nebulosity to a limit onethirtieth that of the quasar light. Optical spectra of three quasars in the present sample-1501+106, 1535+547, and $2209+184$ - have been obtained by Boroson, Oke, and Green (1982). All show the presence of optical continuum and are consistent with the spectra of spiral galaxies.

\section{DISCUSSION}

It is seen from Table 1 that the flux in the near-infrared contributed by the nebulosity within a $10^{\prime \prime}$ diameter diaphragm of the quasar is in some cases a significant fraction of the infrared flux from the quasar itself. The observed $J-K$ colors of the measured nebulosities are shown as a function of redshift in Figure 1. They are compared with the $J-K$ colors of some 19 optically selected galaxies measured by Ellis and Allen (1983). It is seen that within the limited number of objects, the two distributions are the same. Aaronson (1977) has shown that the infrared colors of spiral and elliptical galaxies are very similar; thus the present measurements cannot distinguish between different morphological galaxy types.

The $\mathrm{V}-K$ color of the nebulosity surrounding BL Lac $(2200+420)$ can be obtained by comparing the infrared results with the observations of Thuan, Oke, and Gunn (1975) who measured the $V$ magnitude with a 3 ."7-10" diameter annular diaphragm. If their observed $V$ magnitude, corrected for reddening and the spill from the obscuring disk, is normalized to a $5^{\prime \prime}-10^{\prime \prime}$ annulus, a color of $V-K=3.7 \mathrm{mag}$ is obtained. Although this is redder than most normal galaxies, it is not extreme (Aaronson 1977).

The absolute magnitudes $-M_{J}, M_{H}$, and $M_{K}$ - of the host galaxies were derived assuming a Hubble constant of $50 \mathrm{~km}$ $\mathrm{s}^{-1} \mathrm{Mpc}^{-1}$ and a deceleration parameter, $q_{0}$, of 0.0 . Two corrections, besides those relating directly to the distance factors, were applied to the observed magnitudes listed in Table 1 to bring them to absolute magnitudes: a redshift correction (the $K$-correction) and an estimate of the galaxy flux originating 
TABLE 2

ABsolute Magnitudes

\begin{tabular}{|c|c|c|c|c|c|c|c|}
\hline \multirow[b]{2}{*}{ ОвЈест } & \multirow[b]{2}{*}{$z$} & \multicolumn{2}{|l|}{$1.3 \mu \mathrm{m}$} & \multicolumn{2}{|c|}{$1.65 \mu \mathrm{m}$} & \multicolumn{2}{|c|}{$2.2 \mu \mathrm{m}$} \\
\hline & & $\begin{array}{c}M_{J} \\
\text { (mag) }\end{array}$ & $L_{Q} / L_{G}$ & $\begin{array}{c}M_{H} \\
\text { (mag) }\end{array}$ & $L_{Q} / L_{G}$ & $\begin{array}{c}M_{K} \\
(\mathrm{mag})\end{array}$ & $L_{Q} / L_{G}$ \\
\hline $0026+129$ & 0.142 & $-24.0 \pm 0.3$ & 3.8 & $-24.5 \pm 0.3$ & 3.8 & $-24.5 \pm 0.3$ & 10.8 \\
\hline $0043+039$. & 0.384 & $-24.5 \pm 0.4$ & 6.1 & $-25.1 \pm 0.3$ & 5.8 & $>-24.8$ & $>16.9$ \\
\hline $0838+770$ & 0.131 & $-25.6 \pm 0.3$ & $\ldots$ & $-26.2 \pm 0.3$ & $\ldots$ & $-26.3 \pm 0.3$ & 0.7 \\
\hline $0906+484$. & 0.118 & $-25.0 \pm 0.3$ & & $-25.5 \pm 0.3$ & & $-25.8 \pm 0.3$ & 1.1 \\
\hline $1211+143 .$. & 0.085 & $-22.9 \pm 0.3$ & 8.8 & $-22.3 \pm 0.3$ & 29.4 & $>-23.8$ & $>20.8$ \\
\hline $1351+640$. & 0.087 & $-24.0 \pm 0.3$ & 2.2 & $-24.6 \pm 0.3$ & 2.1 & $-24.8 \pm 0.3$ & 4.3 \\
\hline $1435-067$. & 0.129 & $-23.4 \pm 0.3$ & 2.6 & $-24.0 \pm 0.3$ & 3.0 & $-24.1 \pm 0.3$ & 10.3 \\
\hline $1501+106$. & 0.036 & $-23.6 \pm 0.3$ & 0.8 & $-24.1 \pm 0.3$ & 1.0 & $-24.4 \pm 0.3$ & 2.3 \\
\hline $1535+547$. & 0.038 & $-22.7 \pm 0.3$ & 1.6 & $-23.1 \pm 0.3$ & 2.6 & $-23.5 \pm 0.3$ & 5.4 \\
\hline $1545+210$ & 0.266 & $-25.2 \pm 0.3$ & 1.6 & $-25.7 \pm 0.3$ & 2.2 & $-26.0 \pm 0.3$ & 4.6 \\
\hline $1700+518$. & 0.292 & $-24.4 \pm 0.4$ & 11.3 & $-25.2 \pm 0.4$ & 10.9 & $>-25.5$ & $>29.0$ \\
\hline $1704+608 .$. & 0.371 & $>-25.2$ & $>4.7$ & $-25.0 \pm 0.4$ & 10.2 & $>-25.5$ & $>17.0$ \\
\hline $1718+481 .$. & 1.084 & $>-27.8$ & $>5.6$ & $>-27.7$ & $>8.9$ & $>-28.2$ & $>8.7$ \\
\hline $2200+420 .$. & 0.070 & $-25.1 \pm 0.3$ & 1.0 & $-25.8 \pm 0.3$ & 1.2 & $-26.1 \pm 0.3$ & 2.2 \\
\hline $2209+184$. & 0.070 & $-24.8 \pm 0.3$ & & $-25.3 \pm 0.3$ & & $-25.6 \pm 0.3$ & \\
\hline $2302+029 \ldots$ & 1.044 & $>-26.1$ & $>6.8$ & $-26.0 \pm 0.4$ & 10.8 & $-25.9 \pm 0.3$ & 21.4 \\
\hline
\end{tabular}

outside the annular diaphragm. The derived absolute magnitudes are listed in Table 2.

The $K$-correction applied to the nebulosity was calculated by Persson (1984) for a first-ranked elliptical galaxy with rest frame colors $R-J=2.31 \mathrm{mag}, J-K=1.02 \mathrm{mag}$, and $H-K=0.17 \mathrm{mag}$. The size of this term is typically $0.07,0.01$, and -0.4 mag for the $J, H$, and $K$ bands at the median redshifts $z=0.13$; a tabulation of the $K$-corrections used is given in Table 3. No $K$-correction has been applied to the quasar. This correction is small, however, since for a power-law spectrum the $K$-correction equals $(1+z)^{(1+\alpha)}$, where $\alpha$, the power law of the flux density with frequency, is close to -1 for quasars.

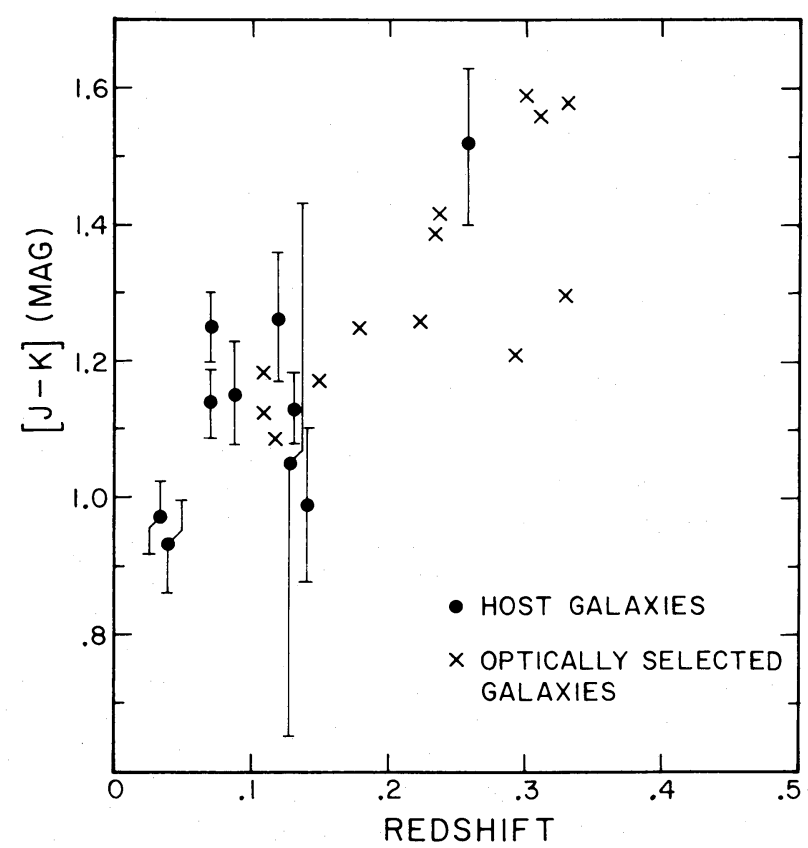

FIG. 1.-The $J-K$ colors of the host galaxies vs. redshift. The optically selected sample used for comparison is from Ellis and Allen (1983). The colors of Ellis and Allen have been transformed to those of the Caltech system using the precepts of Elias et al. (1982).
The correction for the galaxy flux inside the inner annulus radius was estimated through an iterative procedure. The total absolute blue magnitude, $M_{B}$, of the galaxy was estimated assuming that $M_{B}-M_{H}=4 \mathrm{mag}$ (Persson, Frogel, and Aaronson 1979), while the iterative procedure was started assuming an initial absolute $H$ magnitude equaling that measured in the annulus minus 2 mag. The effective radius of the galaxy was then found using the mean relationship for the effective radius as a function of the total blue magnitude as given by Binggeli, Sandage, and Tarenghi (1984) for galaxies in the Virgo cluster or non-Virgo cluster elliptical galaxies. Finally, the contribution within the radius of the annulus was found relative to that measured in the annulus by assuming that the galaxy surface brightness followed the Hubble law (Hubble 1930) and that the peak brightness was centered on the quasar. The core radius was taken as 0.093 the effective radius (Kormendy 1977). The flux thus derived as arising from the galaxy inside the inner annulus radius was typically 3 times that actually observed in the annular diaphragm; the ratio ranged from 1.9 to 3.8 .

TABLE 3

$K$-CORRECTIONS ${ }^{\mathrm{a}}$

\begin{tabular}{|c|c|c|c|}
\hline Redshift & $\begin{array}{c}K(J) \\
(\mathrm{mag})\end{array}$ & $\begin{array}{l}K(H) \\
(\mathrm{mag})\end{array}$ & $\begin{array}{l}K(K) \\
(\mathrm{mag})\end{array}$ \\
\hline 0.00 & 0.00 & 0.00 & 0.00 \\
\hline $0.05 \ldots$ & 0.03 & -0.01 & -0.17 \\
\hline $0.10 .$. & 0.06 & 0.01 & -0.30 \\
\hline $0.15 \ldots \ldots \ldots$ & 0.08 & 0.01 & -0.41 \\
\hline $0.20 \ldots \ldots \ldots$ & 0.10 & 0.01 & -0.51 \\
\hline $0.25 \ldots$ & 0.12 & 0.01 & -0.59 \\
\hline $0.30 \ldots \ldots \ldots$ & 0.11 & 0.02 & -0.65 \\
\hline $0.40 \ldots \ldots \ldots$ & 0.18 & 0.06 & -0.69 \\
\hline $0.50 \ldots \ldots \ldots$ & 0.27 & 0.11 & -0.68 \\
\hline $0.60 \ldots \ldots \ldots$ & 0.31 & 0.13 & -0.67 \\
\hline $0.70 \ldots \ldots \ldots$ & 0.39 & 0.15 & -0.67 \\
\hline $0.80 \ldots \ldots \ldots$ & 0.48 & 0.19 & -0.65 \\
\hline $0.90 \ldots \ldots \ldots$ & 0.55 & 0.24 & -0.62 \\
\hline $1.00 \ldots \ldots \ldots$ & 0.60 & 0.30 & -0.59 \\
\hline $1.10 \ldots \ldots \ldots$ & 0.64 & 0.34 & -0.57 \\
\hline $1.20 \ldots \ldots \ldots$ & 0.69 & 0.40 & -0.55 \\
\hline
\end{tabular}

a Calculated by S. E. Persson 1984 for firstranked elliptical galaxies. 
The contribution to the total flux from the galaxy outside the outer $10^{\prime \prime}$ diameter of the annulus was approximated by assuming the surface brightness $I(r)$, as a function of the radial distance $r$ from the nucleus, was of the form $I(r) \propto I_{0} r^{-2}$. This dependence is in keeping with the optical observations of Gehren et al. (1984) and was assumed to hold out to an outer radius such that the flux arising between the effective radius and the outer radius would be half the total flux of the galaxy. This contribution to the total flux was approximately twice the flux observed in the annulus.

The process of estimating the total flux was repeated iteratively until the final total absolute $H$ magnitude agreed with that initially assumed and the flux outside the effective radius was half the total. The same contributions for the galaxy inside and outside of the annulus were used at all three infrared wavelengths.

The correction for the flux inside a $5^{\prime \prime}$ diameter is consistent with an extrapolation of the mean curve of growth for elliptical and spiral galaxies measured at $2.2 \mu \mathrm{m}$ by Frogel et al. (1978). Numerical tests made with various model parameters show that the solutions with these adopted models are probably uncertain by $\pm 25 \%$. If, however, the surface brightness were proportional to $r^{-1}$, the flux inside the annulus would be equal to, rather than $\sim 3$ times, the flux in the annulus.

For the objects PG $0838+770$, PG $0906+484$, and PG $2209+184$ the model used is probably incorrect since the results imply the galaxy contributes $0.81,0.62$, and 0.73 of the flux within a $5^{\prime \prime}$ diameter diaphragm at $1.3 \mu \mathrm{m}$, and such nebulosity should have removed the sources from the PG sample. In these cases, at least, the surface brightness of the galaxy may not follow the Hubble law, the galaxy may not be centered on the quasar, or multiple nuclei may be present. Also, in cases where the nebulosity contributes much of the flux inside a $5^{\prime \prime}$ diameter diaphragm, the simplistic fit to correct for the spill from the central obscuration into the annulus will be incorrect.

The estimated total galaxy magnitudes are clearly model dependent and quite uncertain. Other models would give different estimates, but whether better estimates would generally be obtained is not a priori certain. The assumptions used probably give too large estimates of the total galaxy flux. It is reassuring that Thuan, Oke, and Gunn (1975) derive a value of $M_{V}=-22.6 \mathrm{mag}$ for $\mathrm{BL}$ Lac implying a corrected color $M_{V}-M_{K}=3.5 \mathrm{mag}$, a color completely consistent with normal galaxies. This may be taken as an indication that the procedures used to estimate the total absolute magnitudes are not too much in error.

The ratios of the infrared luminosity of the quasars to that of the host galaxies $L_{Q} / L_{G}$ are similar to those observed in the blue. In Figure 2 the infrared luminosity ratios at $1.6 \mu \mathrm{m}$ are compared with those obtained by Hutchings et al. (1984) in the blue. The three quasars listed above whose galaxy contribution dominates the flux inside $5^{\prime \prime}$ diameter are arbitrarily excluded from the figure and Table 2. Although the range of the ratio in the visible is perhaps slightly wider than in the infrared, this slight difference can be accounted for by the different techniques. Although the apparent similarity in the two distributions may be a result of various selection effects determining the two samples, it is disturbing. Since the mean $B-H$ color of a wide range of galaxy types lies between 3.5 and 4.1 mag (Persson, Frogel, and Aaronson 1979) while that of quasars is $\sim 2.4 \mathrm{mag}$, the ratio $L_{Q} / L_{G}$ should be systematically lower in the infrared than in the blue by a factor between 3 and 4 . It is encouraging that the two galaxies measured in common with

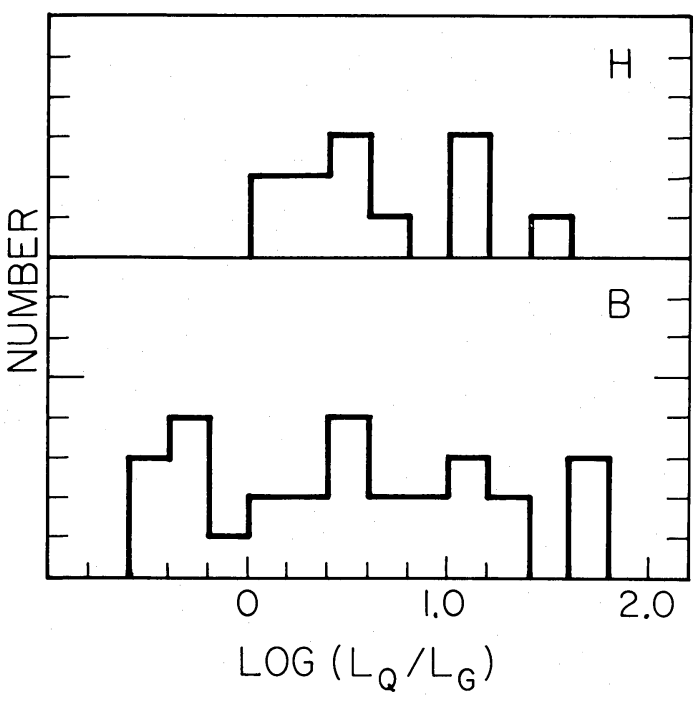

FIG. 2.- Histograms of the ratio of the luminosity ascribed to the host galaxy to that ascribed to the quasar in the $H$ and $B$ wavebands. The three galaxies where the models indicate the galaxy dominates the flux within a $5^{\prime \prime}$ diameter diaphragm have been omitted; see text. The $B$ ratios have been obtained from Hutchings et al.(1984).

Hutchings et al. (1984) have $K$-corrected colors $M_{B}-M_{H}=$ $3.6 \mathrm{mag}$ (PG $0906+484)$ and $3.7 \mathrm{mag}$ (PG 1351+640).

The $K$-corrected colors of the host galaxies, for the subset of galaxies where the colors are well measured, are shown in Table 4. The colors lie close to those measured by Frogel et al. (1978) for E and S0 galaxies, but differ by $\sim 0.1$ mag tending to be bluer in $J-H$ and $J-K$ and correspondingly redder in $H-K$. Although the statistics are not sufficiently understood at this time to warrant drawing major conclusions from them, they may reflect color changes with radial distribution in the galaxies. Note that these colors do not depend upon the correction for the galaxy outside the annular diaphragm.

The absolute magnitudes of the host galaxies, as given in Table 2 and plotted in Figure 3, are typical of bright galaxies, but are certainly not extreme. The distribution of galaxy luminosities is similar in extent to that of normal galaxies $\left(-27<M_{H}<-22 \mathrm{mag}\right)$, but there is an evident bias toward higher luminosities. The median absolute $H$ magnitude is $M_{\mathrm{H}}=-25.4 \mathrm{mag}$. If $M_{B}-M_{H} \approx 3.8 \mathrm{mag}$, the corresponding median absolute $B$ magnitude of the sample is $M_{B}=-21.6$

TABLE 4

K-Corrected Galaxy Colors

\begin{tabular}{cccc}
\hline Object & $\begin{array}{c}M_{J}-M_{H} \\
(\mathrm{mag})\end{array}$ & $\begin{array}{c}M_{H}-M_{K} \\
(\mathrm{mag})\end{array}$ & $\begin{array}{c}M_{J}-M_{K} \\
(\mathrm{mag})\end{array}$ \\
\hline $0026+129 \ldots \ldots \ldots$ & $0.51 \pm 0.13$ & $0.01 \pm 0.12$ & $0.52 \pm 0.12$ \\
$0838+770 \ldots \ldots \ldots$ & $0.57 \pm 0.07$ & $0.11 \pm 0.04$ & $0.68 \pm 0.07$ \\
$0906+484 \ldots \ldots \ldots$ & $0.51 \pm 0.11$ & $0.33 \pm 0.16$ & $0.84 \pm 0.18$ \\
$1351+640 \ldots \ldots \ldots$ & $0.63 \pm 0.09$ & $0.20 \pm 0.09$ & $0.83 \pm 0.11$ \\
$1501+106 \ldots \ldots \ldots$ & $0.56 \pm 0.03$ & $0.26 \pm 0.05$ & $0.83 \pm 0.05$ \\
$1535+547 \ldots \ldots \ldots$ & $0.45 \pm 0.04$ & $0.34 \pm 0.07$ & $0.79 \pm 0.07$ \\
$1545+210 \ldots \ldots \ldots$ & $0.47 \pm 0.12$ & $0.32 \pm 0.16$ & $0.78 \pm 0.17$ \\
$2200+420 \ldots \ldots \ldots$ & $0.68 \pm 0.08$ & $0.33 \pm 0.06$ & $1.01 \pm 0.08$ \\
$2209+184 \ldots \ldots \ldots$ & $0.50 \pm 0.05$ & $0.35 \pm 0.04$ & $0.85 \pm 0.06$ \\
Mean $\ldots \ldots \ldots \ldots$ & $0.54 \pm 0.08$ & $0.25 \pm 0.12$ & $0.79 \pm 0.13$ \\
E and $\mathrm{S}^{\mathrm{a}} \ldots \ldots \ldots$. & $0.68 \pm 0.04$ & $0.20 \pm 0.03$ & $0.88 \pm 0.05$ \\
\hline
\end{tabular}

a From Frogel et al. 1978. 


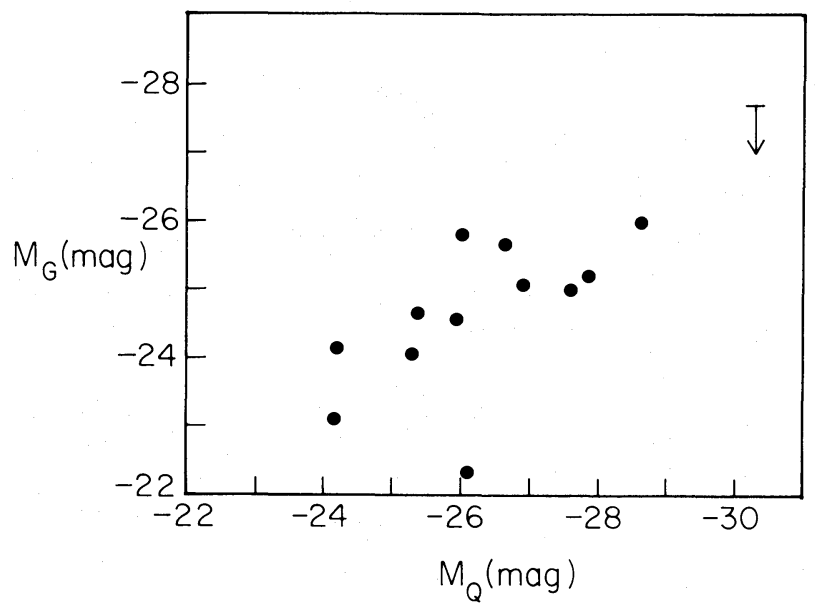

Fig. 3.-The absolute magnitudes at $H$ of the host galaxies plotted against that of the quasars. The uncertainties are typically assumed to be of order 0.3 mag. The three galaxies where the models indicate the galaxy dominates the flux within a 5 " diameter diaphragm have been omitted; see text. The figure is similar to Fig. 7 of Hutchings et al. (1984). The limit is for the galaxy PG $1718+481$ and represents a $3 \sigma$ limit. The actual measurement was $2.4 \sigma$.

mag, brighter than typical field or cluster galaxies (e.g., Schechter 1976).

Figure 3, the counterpart to Figure 7 of Hutchings et al. (1984), shows that the correlation, including the slope, between host galaxy and quasar luminosity previously noted in the $B$ waveband extends to the infrared. Selection effects are clearly important in considering the total range of absolute flux densities associated with the nebulosity. As noted by Hutchings et al., the top left-hand area of the diagram is empty since galaxies lying here would have caused the source to be classified as a
Seyfert 1 galaxy rather than a quasar, and the source would not have been included in the sample. The absence of sources in the bottom right-hand area suggests a lower bound on the ratio of host galaxy to quasar luminosities, strengthening the conjecture made by Hutchings et al. It is interesting that none of the measurements except PG $1718+481$ resulted in less than $3 \sigma$ limits at $1.65 \mu \mathrm{m}$, the most sensitive infrared wavelength for detecting nebulosity, when the observations over several nights were averaged; see Table 2 . The detection of nebulosity around PG $1718+481$ was at the $2.4 \sigma$ level. Nebulosity at $B$ was not detected around PG $1700+518$ by Hutchings et al. (1984) to a limit corresponding to only $M_{B}-M_{H}>2.8 \mathrm{mag}$. Further observations of higher luminosity quasars are needed to see whether there are a significant number of faint galaxies hosting quasars.

\section{CONCLUSIONS}

Measurements of the near-infrared flux densities of the nebulosity around several quasars have shown that in some quasars the nebulosity within $10^{\prime \prime}$ diameter contributes a significant fraction of the near-infrared flux of the quasar. The observations are consistent with the host galaxies being normal elliptical or spiral galaxies. The galaxies have luminosities similar to those observed from normal galaxies at the redshifts of the quasars, but are on the bright end of the distribution.

We thank S. E. Persson for providing us with the $K$ corrections and many stimulating discussions. We thank the staff of the Palomar Observatory for their help, and especially Juan Carrasco who has been our night assistant on the 200 inch $(5 \mathrm{~m})$ telescope. This work was supported by a grant from NSF.
Aaronson, M. 1977, Ph.D. thesis, Harvard University.

Balick, B., and Heckman, T. 1963, Ap. J. (Letters), 265, L1.

Binggeli, B., Sandage, A., and Tarenghi, M. 1984, A.J., 89, 64.

Boroson, T. A., and Oke, J. B. 1982, Nature, 296, 397.

. 1984, Ap.J., 281, 535.

Boroson, T. A., Oke, J. B., and Green, R. 1982, Ap, J, 263, 32

Elias, J. H., Frogel, J. A., Matthews, K., and Neugebauer, G. 1982, A.J., 87, 1029.

Ellis, R. S., and Allen, D. A. 1983, M.N.R.A.S., 203, 685.

Frogel, J. A., Persson, S. E., Aaronson, M., and Matthews, K. 1978, Ap. J., 220, 75 .

Gehren, T., Fried, J., Wehinger, P. A., and Wyckoff, S. 1984, Ap. J., 278, 11.

Hubble, E. 1930, Ap. J., 71, 231.

Hutchings, J. B., Crampton, D., and Campbell, B. 1984, Ap. J., 280, 41.

\section{REFERENCES}

Hutchings, J. B., Crampton, D., Campbell, B., Duncan, D., and Glendenning, B. 1984, Ap. J.Suppl., 55, 319.

Kormendy, J. 1977, Ap. J., 218, 333.

Malkan, M. A., Margon, B., and Chanan, G. A. 1984, Ap. J., 280, 66.

Neugebauer, G., Oke, J. B., Becklin, E. E., and Matthews, K. 1979, Ap. J., 230 , 79.

Persson, S. E. 1984, private communication.

Persson, S. E., Frogel, J. A., and Aaronson, M. 1979, Ap. J. Suppl., 39, 61.

Schechter, P. 1976, Ap. J., 203, 297.

Schmidt, M., and Green, R. 1983, Ap. J., 269, 353

Stockton, A. 1982, Ap. J., 257, 33.

Thuan, T. X., Oke, J. B., and Gunn, J. E. 1975, Ap. J., 201, 45.

Tyson, J. A., Baum, W. A., and Kreidl, T. 1982, Ap. J. (Letters), 257, L1.

Wyckoff, S., Wehinger, P., and Gehren, T. 1981, Ap. J., 247, 750.

J. H. Elias, K. Matthews, G. Neugebauer, and B. T. Soifer: Downs Laboratory of Physics 320-47, California Institute of Technology, Pasadena, CA 91125 